\title{
Appendix A \\ THE ATOMIC PHYSICS OF OXYGEN
}

\section{A.1 Introduction}

In this Appendix, we review the status of the atomic data sets required for handling oxygen plasma diagnostics and abundance determinations. These have been calculated and measured for several decades by a group of experienced and dedicated scientists who are determined to attain the data integrity and accuracy indispensable for astrophysical applications. In a long and arduous process, periodic revisions have certainly resulted in significant improvements but also in a few unhappy surprises.

As scientific research rapidly becomes very data intensive, atomic and molecular data activities in the field of laboratory astrophysics are reaching unprecedented levels and beginning to secure more adequate funding. We would just like to mention two recent initiatives: (i) the Virtual Atomic and Molecular Data Center (VAMDC ${ }^{21}$ - Dubernet et al., 2010; Rixon et al., 2011), a large multinational consortium aimed at integrating, normalising and modernising at least 20 atomic and molecular databases into a distributed virtual warehouse; and (ii) AstroAtom ${ }^{22}$, a blog which, by means of Web 2.0 tools, attempts to consolidate the atomic data user-producer community in astrophysics by promoting scientific exchange before formal publication.

In this report we concentrate on collisionally excited lines (Sect. A.2), but also discuss recent advances in the study of optical recombination lines (Sect. A.3), charge transfer (Sect. A.4), K-shell photoabsorption (Sect. A.5) and opacities (Sect. A.6). Whenever possible, the assessed and recommended data are organised in spreadsheets (labelled Ion.datatype where Ion is the file name and datatype the corresponding tab name) which are publicly available online as supplementary data from Google Docs ${ }^{23}$. This handy atomic database will be updated regularly and managed through subsequent releases, the initial version being referred to as V1. Statistical data-set comparisons are mainly carried out in order to give general

\footnotetext{
${ }^{21}$ http: //www. vamdc.eu/

${ }^{22}$ https://astroatom. wordpress.com/

${ }^{23}$ http: //bit.1y/pUwMEf
} 
accuracy ratings, but more specific estimates may be obtained for desired items by looking through these comparisons in the spreadsheets.

\section{A.2 Collisionally excited lines}

The radiative and collisional rates considered in this review and required in the interpretation of collisionally excited lines have been computed with a suite of stateof-the-art atomic physics codes that address two important issues: configuration interaction and relativistic effects. Radiative rates ( $A$-values) are mainly obtained with multi-configuration atomic structure codes that include the relativistic corrections with a Breit-Pauli Hamiltonian, namely SUPERSTRUCTURE (Eissner et al. 1974), CIV3 (Hibbert 1975), HFR (Cowan 1981), MCHF (Froese Fischer et al. 1997, 2007) and AUTOSTRUCTURE (Badnell 2011), and with the GRASP general purpose Dirac-Fock atomic structure package (Grant et al. 1980).

Electron collision rates for transitions between two atomic levels, $i$ and $j(j>$ $i)$, can be expressed in $\mathrm{cm}^{3} \mathrm{~s}^{-1}$ as

$$
q(j, i ; T)=\frac{8.631 \times 10^{-6} \Upsilon(j, i ; T)}{\omega(j) T^{1 / 2}}
$$

and

$$
q(i, j)=\frac{\omega(j)}{\omega(i)} \exp \left[-\frac{\Delta E(i, j)}{\kappa T}\right] q(j, i)
$$

where $\omega(j)$ and $\omega(i)$ are the statistical weights of the upper and lower levels, respectively, $\Delta E(i, j)$ is the level energy separation, $T$ is the electron temperature in $\mathrm{K}$ and $\kappa$ is the Boltzmann constant. The dimensionless effective collision strength $\Upsilon(j, i ; T)$ is obtained by integrating the collision strength $\Omega(i, j ; E)$ over a Maxwellian distribution

$$
\Upsilon(j, i ; T)=\int_{0}^{\infty} \Omega(i, j ; E) \exp \left(-E_{j} / \kappa T\right) \mathrm{d}\left(E_{j} / \kappa T\right) .
$$

We consider here collision strengths and, hence, effective collision strengths that are mainly calculated with the BPRM close-coupling (multichannel) Breit-Pauli $R$-matrix package (Berrington et al. 1978; Scott \& Burke 1980; Scott \& Taylor 1982), the intermediate-coupling frame transformation (ICFT) $R$-matrix method (Griffin et al. 1998) and the DARC Dirac Atomic $R$-matrix Code of P.H. Norrington and I.P. Grant (private communication).

We now proceed to evaluate for each $\mathrm{O}$ ion the atomic models that have been proposed and the data sets that are currently available. It will be seen that considerable efforts have been made to implement multi-level models and wide temperature ranges in order to cater for most astronomical plasma conditions.

\section{A.2.1 O I}

We consider for $\mathrm{O}$ I the 23-level fine-structure model (01.ElevIC) computed by Tachiev \& Froese Fischer (2002) with MCHF including extensive configuration 
Table A.1. $A$-value ratios for forbidden lines in the ground configuration of $\mathrm{O}$ ions.

\begin{tabular}{llllll}
\hline \hline Ion & Parameter & TFF & GMZ & SZ & Z \\
\hline O I & $A\left({ }^{1} \mathrm{D}_{2}-{ }^{3} \mathrm{P}_{2}\right)$ & $6.503 \mathrm{E}-3$ & $6.535 \mathrm{E}-3$ & $6.446 \mathrm{E}-3$ & \\
& $A\left({ }^{1} \mathrm{D}_{2}-{ }^{3} \mathrm{P}_{1}\right)$ & $2.101 \mathrm{E}-3$ & $2.111 \mathrm{E}-3$ & $2.151 \mathrm{E}-3$ & \\
& Ratio & 3.10 & 3.10 & 3.00 & \\
& $A\left({ }^{1} \mathrm{~S}-{ }^{1} \mathrm{D}\right)$ & $1.260 \mathrm{E}+0$ & $1.124 \mathrm{E}+0$ & & \\
& $A\left({ }^{1} \mathrm{~S}-{ }^{3} \mathrm{P}\right)$ & $7.877 \mathrm{E}-2$ & $7.940 \mathrm{E}-2$ & & $3.588 \mathrm{E}-5$ \\
& Ratio & 16.0 & 14.2 & & $1.810 \mathrm{E}-4$ \\
O II & $A\left({ }^{2} \mathrm{D}_{5 / 2}-{ }^{4} \mathrm{~S}_{3 / 2}^{\mathrm{o}}\right)$ & $4.123 \mathrm{E}-5$ & & & \\
& $A\left({ }^{2} \mathrm{D}_{3 / 2}-{ }^{4} \mathrm{~S}_{3 / 2}^{\mathrm{o}}\right)$ & $1.635 \mathrm{E}-4$ & & & \\
& Ratio & 0.252 & & & \\
O III & $A\left({ }^{1} \mathrm{D}_{2}-{ }^{3} \mathrm{P}_{2}\right)$ & $2.028 \mathrm{E}-2$ & $2.041 \mathrm{E}-2$ & $2.046 \mathrm{E}-2$ & \\
& $A\left({ }^{1} \mathrm{D}_{2}-{ }^{3} \mathrm{P}_{1}\right)$ & $6.951 \mathrm{E}-3$ & $6.995 \mathrm{E}-3$ & $6.791 \mathrm{E}-3$ & \\
& Ratio & 2.92 & 2.92 & 3.01 & \\
\hline
\end{tabular}

TFF: Tachiev \& Froese Fischer (2001, 2002). GMZ: Galavís et al. (1997). SZ: Storey \& Zeippen (2000). Z: Zeippen (1987).

interaction and relativistic Breit-Pauli corrections, which yields theoretical level energies within $1 \%$ of the spectroscopic values. Regarding the $A$-values for the important forbidden transitions within the ground $2 \mathrm{~s}^{2} 2 \mathrm{p}^{4}$ configuration, a comparison (01.Aval2p4) of the values obtained in this model with those computed by Galavís et al. (1997) with SUPERSTRUCTURE using a similar approximation results in good agreement: better than $10 \%$ for the magnetic dipole (M1) and $15 \%$ for the electric quadrupole (E2) transitions. It is worth mentioning that both $A$-value data sets are computed with the experimental level energy separations $\Delta E(i, j)$.

Storey \& Zeippen (2000) have shown that the astrophysically important $A\left({ }^{1} \mathrm{D}_{2}-\right.$ $\left.{ }^{3} \mathrm{P}_{2}\right) / A\left({ }^{1} \mathrm{D}_{2}-{ }^{3} \mathrm{P}_{1}\right)$ branching ratio is sensitive to the Breit-Pauli corrections to the M1 operator which have been neglected by both Tachiev \& Froese Fischer (2002) and Galavís et al. (1997). Although the differences are small (see Table A.1), they are nevertheless relevant in plasma diagnostics and instrument calibrations; for instance, Sharpee \& Slanger (2006) have estimated this ratio for O I at 2.997(16) from observations in the terrestrial nightglow which, as shown in Table A.1, is in excellent agreement with the value of 3.00 determined by Storey \& Zeippen (2000).

A less satisfactory situation arises in a comparison of the theoretical $A\left({ }^{1} \mathrm{~S}-\right.$ $\left.{ }^{1} \mathrm{D}\right) / A\left({ }^{1} \mathrm{~S}-{ }^{3} \mathrm{P}\right)$ ratio with those measured in the laboratory and observed in the terrestrial nightglow (Slanger et al. 2006). As seen in Table A.1, there is a $13 \%$ difference between the theoretical ratios which can be attributed to configuration interaction and ion model sensitivity in E2 transitions, but the atmospheric observations result in a consistent value of 9.8(10) while all laboratory measurements are above 18.6. The observed value has been recently confirmed in the auroral spectrum at 9.3(5) by Gattinger et al. (2009). It is difficult to agree with the assertion by Slanger et al. (2006) that the theoretical $A\left({ }^{1} \mathrm{~S}-{ }^{3} \mathrm{P}\right)$ is underestimated since the accord in Table A.1 for this transition is within 1\%. Therefore, these 
discrepancies between theory, laboratory and observations have not as yet been resolved and have consequences for auroral and dayglow processes.

Tachiev \& Froese Fischer (2002) also provide $A$ - and $f$-values for allowed transitions that are listed in 01 . AvalIC, their $f$-values being within $10 \%$ of those computed by Hibbert et al. (1991) including configuration interaction and small energy corrections to the diagonal matrix elements, and by Tayal (2009) using a Breit-Pauli B-spline box-based $R$-matrix method.

Fine-structure effective collision strengths for electron impact have been computed by Bell et al. (1998) for transitions within the $2 \mathrm{~s}^{2} 2 \mathrm{p}^{4}{ }^{3} \mathrm{P}$ ground term of O I (see 01 .EColStIC). The $R$-matrix method is used with a close-coupling expansion that includes pseudo-states to account for the dipole polarisability of the ${ }^{3} \mathrm{P}$ term, and term-energy corrections are introduced in the inner-region Hamiltonian before diagonalisation to match the spectroscopic splittings. Due to a careful study of the near-threshold behaviour of the cross section, significant differences (factors of 2 to 4 ) have been found with respect to previous estimates thus complying with ionospheric requirements.

Effective collision strengths have also been computed in $L S$ coupling by Zatsarinny \& Tayal (2003) with the B-spline $R$-matrix method for transitions involving terms of the ground and excited configurations which are listed in 01. EColStLS. In order to ensure proper convergence of the close-coupling expansion, a large target has been used with 26 terms comprising both bound and autoionising states represented by non-orthogonal orbitals that leads to a significant reduction of undesirable pseudo-resonances.

A more recent 38-state $R$-matrix calculation has been performed by Barklem (2007) to obtain excitation rates for NLTE modelling of oxygen in late-type stellar atmospheres. It excludes autoionising states and polarised pseudo-states in the close-coupling expansion, and the excitation cross sections are found to be plagued by pseudo-resonances that are somehow smoothed out, finding reasonable agreement (within a factor of 3) with Zatsarinny \& Tayal (2003). In the light of these comparisons, we would rate the effective collision strengths listed by the latter as better than a factor of 2 .

\section{A.2.2 O II}

For the $\mathrm{O}$ II atomic representation, we recommend the 62-level fine-structure model of Tachiev \& Froese Fischer (2002) computed with MCHF including a Breit-Pauli Hamiltonian and extensive configuration interaction (see 02.Elev). The agreement of the theoretical level energies with spectroscopic measurements is better than $1 \%$. We also consider the smaller 47-level target representations adopted in the electron impact excitation calculation of Tayal (2007), where level energies and $f$-values are computed with MCHF using non-orthogonal (Target $\mathrm{A}$ ) and orthogonal (Target B) orbitals. As described by Tayal (2007), Target A is in better accord (within 2\%) with the spectroscopic level energies than Target B (5\%).

As shown in 02.Aval2p3, the $A$-values listed by Tachiev \& Froese Fischer (2002) for the forbidden transitions within the $2 \mathrm{~s}^{2} 2 \mathrm{p}^{3}$ ground configuration are 
in good agreement (5\%) with the earlier work by Zeippen (1987) except for the topical ${ }^{2} \mathrm{D}_{J}^{\mathrm{o}}-{ }^{4} \mathrm{~S}_{3 / 2}^{\mathrm{o}}$ doublet that is used in density diagnostics. It is well known that the M1 $A$-values for the half-filled configuration are particularly sensitive to the Breit-Pauli corrections to the transition operator, which have been neglected by Tachiev \& Froese Fischer (2002) and thus lead to a 30\% discrepant branching ratio (see Table A.1). We therefore recommend the $A$-values by Zeippen (1987).

Tachiev \& Froese Fischer (2002) also list $A$ - and $f$-values for allowed lines (see 02. AvalE1E2) which can be compared with the data sets by Bell et al. (1994) computed with the CIV3 code and by Tayal (2007). A-values in Bell et al. (1994) for transitions with $\log A \geq 7$ agree to within $25 \%$, which may be considered satisfactory, while discrepancies as large as a factor of 2 are found in Tayal (2007).

The computational estimates of the effective collision strengths for transitions within the ground configuration of $\mathrm{O}$ II have seen interesting developments since the 5-state $(L S)$ close-coupling calculation of Pradhan (1976). Twenty years later, McLaughlin \& Bell (1998) computed new data in an 11-level (fine structure) BreitPauli $R$-matrix approximation, finding large differences that were attributed to their extended close-coupling expansion and increased resolution of the resonance structure. Moreover, the new results questioned the validity of the low-density scaling of the ${ }^{2} \mathrm{D}_{J}^{\mathrm{o}}-{ }^{4} \mathrm{~S}_{3 / 2}^{\circ}$ doublet intensity ratio, assumed to be equal to the ratio of the statistical weights of the upper levels (Seaton \& Osterbrock 1957), and were eventually shown to be inconsistent with observations in planetary nebulae (Copetti \& Writzl 2002; Wang et al. 2004). Subsequent close-coupling calculations (Kisielius et al. 2009; Montenegro et al. 2006; Pradhan et al. 2006; Tayal 2006a, 2007) have confirmed the inaccuracies of McLaughlin \& Bell (1998).

In our opinion, Kisielius et al. (2009) have carried out a conclusive analysis of this problem by performing a Breit-Pauli $R$-matrix calculation with various wavefunction expansions and target orbitals, and using both ab initio and experimental target-level energy separations and very fine energy meshes (02.EColSt2p3). They have found reasonable agreement (a few percent) with the results by Montenegro et al. (2006) and Tayal (2007), but for some transitions, e.g. the ${ }^{2} \mathrm{D}_{3 / 2}^{\mathrm{o}}{ }^{2}{ }^{2} \mathrm{D}_{5 / 2}^{\mathrm{o}}$ (see Fig. A.1), noticeable discrepancies are encountered at low temperatures $(36 \%$ and $25 \%$, respectively). These are believed to be due to variations of the resonance pattern located just above the transition threshold which is sensitive to the positions of the target-level energies in the calculation, i.e. whether theoretical or experimental values are chosen.

Effective collision strengths for transitions to excited configurations are given in Tayal (2007) (see 02.EColSt). As pointed out by Kisielius et al. (2009) and confirmed by S.S. Tayal (private communications), levels 4 and 5 in Table 3 of Tayal (2007) should be interchanged in order to retrieve the correct data.

\section{A.2.3 O III}

The situation of the atomic parameters for O III is on firmer ground than that for O I although it shows some of the problems that have been previously discussed. 


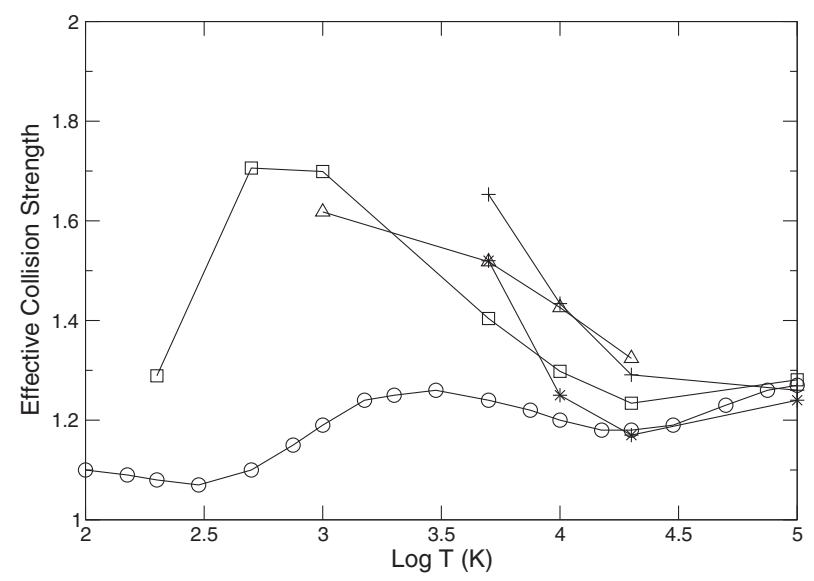

Fig. A.1. Effective collision strength for the ${ }^{2} \mathrm{D}_{3 / 2}^{\mathrm{o}}-{ }^{2} \mathrm{D}_{5 / 2}^{\mathrm{o}}$ transition in $\mathrm{O}$ II showing low-temperature sensitivity to the resonance structure. Circles: Kisielius et al. (2009) using experimental target-level energy separations. Squares: Kisielius et al. (2009) with theoretical target-level energy separations. Triangles: Montenegro et al. (2006). Crosses: Tayal (2007). Stars: McLaughlin \& Bell (1998).

We consider the 46-level atomic models computed by Tachiev \& Froese Fischer (2001) with the Breit-Pauli configuration-interaction MCHF code, which reproduces the measured level energies to within 1\%, and by Aggarwal \& Keenan (1999) with CIV3. The latter model is mainly tailored for the collisional calculation and does not include relativistic effects (see 03.Elev).

The radiative data for $\mathrm{O}$ III have been extensively analysed by Froese Fischer et al. (2009). As shown in 03. Aval2p2, A-values computed by Tachiev \& Froese Fischer (2001) for the forbidden transitions within the $2 \mathrm{~s}^{2} 2 \mathrm{p}^{2}$ ground configuration agree fairly well with those by Galavís et al. (1997), the largest discrepancy being around $25 \%$ for an E2 transition with a small $\left(\sim 10^{-6} \mathrm{~s}^{-1}\right) A$-value. Their estimated lifetimes for the ${ }^{1} \mathrm{~S}$ level, $523 \mathrm{~ms}$ and $559 \mathrm{~ms}$ respectively, are in very good agreement with two measurements: 530(25) ms (Träbert et al. 2000) and 540(27) ms (Smith et al. 2004). The corrections to the M1 transitions pointed out by Storey \& Zeippen (2000) must be nevertheless taken into account as they have a noticeable impact on the astrophysically important $A\left({ }^{1} \mathrm{D}_{2}-{ }^{3} \mathrm{P}_{2}\right) / A\left({ }^{1} \mathrm{D}_{2}-{ }^{3} \mathrm{P}_{1}\right)$ branching ratio (see Table A.1).

$A$-values listed by Tachiev \& Froese Fischer (2001) for E1 transitions (03.AvalE1) can be compared with those computed by Aggarwal et al. (1997) who took into account extensive configuration interaction and relativistic effects with the code CIV3. For transitions with $\log A \geq 7$, the agreement is found to be better than $30 \%$ if the 11 discrepant transitions listed in Table A.2 are excluded. This rating is corroborated in a comparison of the theoretical lifetimes for the $2 \mathrm{~s} 2 \mathrm{p}^{3}{ }^{5} \mathrm{~S}_{2}^{\text {o }}$ level, $1.24 \mathrm{~ms}$ and $1.72 \mathrm{~ms}$ respectively, with the measurement of $1.250(13) \mathrm{ms}$ by Träbert et al. (2000) in a heavy-ion storage ring. 
Table A.2. Discrepant $A$-values for E1 transitions in O III.

\begin{tabular}{llll}
\hline \hline Upper level & Lower level & AHK & TFF \\
\hline $2 \mathrm{~s}^{2} 2 \mathrm{p} 3 \mathrm{p}{ }^{1} \mathrm{P}_{1}$ & $2 \mathrm{~s}^{2} 2 \mathrm{p} 3 \mathrm{~s}^{1} \mathrm{P}_{1}^{\mathrm{o}}$ & $9.27 \mathrm{E}+6$ & $3.09 \mathrm{E}+7$ \\
$2 \mathrm{~s}^{2} 2 \mathrm{p} 3 \mathrm{p}{ }^{3} \mathrm{P}_{0}$ & $2 \mathrm{~s} 2 \mathrm{p}^{3}{ }^{3} \mathrm{P}_{1}^{\mathrm{o}}$ & $2.94 \mathrm{E}+7$ & $1.30 \mathrm{E}+7$ \\
$2 \mathrm{~s}^{2} 2 \mathrm{p} 3 \mathrm{~d}{ }^{3} \mathrm{~F}_{2}^{\mathrm{o}}$ & $2 \mathrm{~s}^{2} 2 \mathrm{p} 3 \mathrm{p}{ }^{1} \mathrm{P}_{1}$ & $3.31 \mathrm{E}+7$ & $6.06 \mathrm{E}+7$ \\
$2 \mathrm{~s}^{2} 2 \mathrm{p} 3 \mathrm{~d}{ }^{3} \mathrm{~F}_{3}^{\mathrm{o}}$ & $2 \mathrm{~s}^{2} 2 \mathrm{p}^{2}{ }^{3} \mathrm{P}_{2}$ & $1.71 \mathrm{E}+7$ & $4.08 \mathrm{E}+7$ \\
$2 \mathrm{~s}^{2} 2 \mathrm{p} 3 \mathrm{~d}{ }^{1} \mathrm{D}_{2}^{\mathrm{o}}$ & $2 \mathrm{~s}^{2} 2 \mathrm{p}^{2}{ }^{3} \mathrm{P}_{1}$ & $1.40 \mathrm{E}+7$ & $3.45 \mathrm{E}+7$ \\
$2 \mathrm{~s}^{2} 2 \mathrm{p} 3 \mathrm{~d}{ }^{1} \mathrm{D}_{2}^{\mathrm{o}}$ & $2 \mathrm{~s}^{2} 2 \mathrm{p} 3 \mathrm{p}^{3} \mathrm{D}_{1}$ & $2.80 \mathrm{E}+7$ & $5.54 \mathrm{E}+7$ \\
$2 \mathrm{~s}^{2} 2 \mathrm{p} 3 \mathrm{~d}{ }^{3} \mathrm{P}_{2}^{\mathrm{o}}$ & $2 \mathrm{~s}^{2} 2 \mathrm{p}^{2}{ }^{1} \mathrm{D}_{2}$ & $6.36 \mathrm{E}+6$ & $1.02 \mathrm{E}+7$ \\
$2 \mathrm{~s}^{2} 2 \mathrm{p} 3 \mathrm{~d}{ }^{3} \mathrm{P}_{1}^{\mathrm{o}}$ & $2 \mathrm{~s}^{2} 2 \mathrm{p} 3 \mathrm{p}{ }^{3} \mathrm{P}_{1}$ & $1.31 \mathrm{E}+7$ & $2.19 \mathrm{E}+7$ \\
$2 \mathrm{~s}^{2} 2 \mathrm{p} 3 \mathrm{~d}{ }^{1} \mathrm{P}_{1}^{\mathrm{o}}$ & $2 \mathrm{~s}^{2} 2 \mathrm{p}^{2}{ }^{3} \mathrm{P}_{0}$ & $3.99 \mathrm{E}+6$ & $1.57 \mathrm{E}+7$ \\
$2 \mathrm{~s}^{2} 2 \mathrm{p} 3 \mathrm{~d}{ }^{1} \mathrm{P}_{1}^{\mathrm{o}}$ & $2 \mathrm{~s}^{2} 2 \mathrm{p}^{2}{ }^{1} \mathrm{D}_{2}$ & $7.75 \mathrm{E}+8$ & $2.86 \mathrm{E}+8$ \\
$2 \mathrm{~s}^{2} 2 \mathrm{p} 3 \mathrm{~d}{ }^{1} \mathrm{P}_{1}^{\mathrm{o}}$ & $2 \mathrm{~s}^{2} 2 \mathrm{p} 3 \mathrm{p}{ }^{1} \mathrm{~S}_{0}$ & $1.92 \mathrm{E}+7$ & $3.45 \mathrm{E}+7$ \\
\hline
\end{tabular}

AHK: Aggarwal et al. (1997). TFF: Tachiev \& Froese Fischer (2001).

Electron impact excitation rates for carbon-like ions, including O III, has been previously assessed by Monsignori Fossi \& Landini (1994). More recently, effective collision strengths have been computed for $\mathrm{O}$ III with the $R$-matrix method by Aggarwal \& Keenan (1999) for the 46-level atomic model selected above using a 26term $L S$ target. As specified by Aggarwal \& Keenan (1999), data for the missing transitions in their original Tables $4 \mathrm{~A}-\mathrm{B}$, which are reproduced in 03. EColSt, can be directly determined from statistical weight ratios.

Aggarwal \& Keenan (1999) have compared their data set with previous computations (Aggarwal 1983, 1985, 1993; Aggarwal \& Hibbert 1991) finding large discrepancies (up to a factor of 3 ) for some transitions at both low and high energies, which are attributed to pseudo-resonances, non-converged partial wave expansions, inadequate energy ranges and, in some cases, to unexplained reasons. However, for transitions within the ground configuration, there is good agreement with the $R$-matrix calculation by Lennon \& Burke (1994) (well within 10\%) and, for the specific ${ }^{3} \mathrm{P}-{ }^{1} \mathrm{D}$ transition, with the absolute experimental cross section by Niimura et al. (2002).

\section{A.2.4 O IV}

We favour the 75-level atomic model computed by Aggarwal \& Keenan (2008a) with the GRASP multi-configuration Dirac-Fock package (see 04.Elev). Two approximations are considered: with (AK08b) and without (AK08a) the Breit and quantum electrodynamic (QED) corrections. Energies computed for 23 levels by Tachiev \& Froese Fischer (2000), for 20 levels by Corrégé \& Hibbert (2004) and for 54 levels by Tayal (2006b) with different Breit-Pauli configuration-interaction methods are also tabulated. The comparison for 68 spectroscopic levels is in general very good, AK08b being on average in better accord (3\%) than AK08a (5\%) while values by Tachiev \& Froese Fischer (2000), Corrégé \& Hibbert (2004) and 
Table A.3. $A$-values for forbidden and intercombination transitions in O IV.

\begin{tabular}{llllll}
\hline \hline Upper level & Lower level & GMZ & TFF & $\mathrm{CH}$ & $\mathrm{AK}$ \\
\hline $2 \mathrm{~s}^{2} 2 \mathrm{p}{ }^{2} \mathrm{P}_{3 / 2}^{\mathrm{o}}$ & $2 \mathrm{~s}^{2} 2 \mathrm{p}{ }^{2} \mathrm{P}_{1 / 2}^{\mathrm{o}}$ & $5.17 \mathrm{E}-4$ & $5.22 \mathrm{E}-4$ & & $4.61 \mathrm{E}-4$ \\
$2 \mathrm{~s} 2 \mathrm{p}^{2}{ }^{4} \mathrm{P}_{1 / 2}$ & $2 \mathrm{~s}^{2} 2 \mathrm{p}{ }^{2} \mathrm{P}_{1 / 2}^{\mathrm{o}}$ & $1.72 \mathrm{E}+3$ & $1.49 \mathrm{E}+3$ & $1.47 \mathrm{E}+3$ & $9.42 \mathrm{E}+2$ \\
$2 \mathrm{~s} 2 \mathrm{p}^{2}{ }^{4} \mathrm{P}_{1 / 2}$ & $2 \mathrm{~s}^{2} 2 \mathrm{p}{ }^{2} \mathrm{P}_{3 / 2}^{\mathrm{o}}$ & $1.76 \mathrm{E}+3$ & $1.47 \mathrm{E}+3$ & $1.45 \mathrm{E}+3$ & $1.01 \mathrm{E}+3$ \\
$2 \mathrm{~s} 2 \mathrm{p}^{2}{ }^{4} \mathrm{P}_{3 / 2}$ & $2 \mathrm{~s}^{2} 2 \mathrm{p}{ }^{2} \mathrm{P}_{1 / 2}^{\mathrm{o}}$ & $4.84 \mathrm{E}+1$ & $3.91 \mathrm{E}+1$ & $3.75 \mathrm{E}+1$ & $2.31 \mathrm{E}+1$ \\
$2 \mathrm{~s} 2 \mathrm{p}^{2}{ }^{4} \mathrm{P}_{3 / 2}$ & $2 \mathrm{~s}^{2} 2 \mathrm{p}^{2} \mathrm{P}_{3 / 2}^{\mathrm{o}}$ & $3.15 \mathrm{E}+2$ & $2.94 \mathrm{E}+2$ & $2.90 \mathrm{E}+2$ & $1.44 \mathrm{E}+2$ \\
$2 \mathrm{~s} 2 \mathrm{p}^{2}{ }^{4} \mathrm{P}_{5 / 2}$ & $2 \mathrm{~s}^{2} 2 \mathrm{p}{ }^{2} \mathrm{P}_{3 / 2}^{\mathrm{o}}$ & $1.33 \mathrm{E}+3$ & $1.19 \mathrm{E}+3$ & $1.16 \mathrm{E}+3$ & $6.54 \mathrm{E}+2$ \\
\hline
\end{tabular}

GMZ: Galavís et al. (1998). TFF: Tachiev \& Froese Fischer (2000). CH: Corrégé \& Hibbert (2004). AK: Aggarwal \& Keenan (2008a).

Tayal (2006b) are well within $1 \%$. In some of the theoretical data sets, inverted fine-structure splittings are found for some of the terms (mainly those with $L=2$ ).

All these calculations list $A$-values which enable a detailed comparison (see 04.Aval). In Table A.3, we tabulate $A$-values for the astrophysically relevant forbidden and intercombination transitions within the $n=2$ complex. The agreement between Tachiev \& Froese Fischer (2000) and Corrégé \& Hibbert (2004) is outstanding $(5 \%)$ while larger differences are encountered in the data by Galavís et al. (1998) (20\%) and Aggarwal \& Keenan (2008a) (a factor of 2).

Regarding the allowed transitions within $n=2$, it may be seen in Figure A.2 that, with respect to the $A$-values listed by Tachiev \& Froese Fischer (2000), the agreement with Corrégé \& Hibbert (2004) is excellent (well within 5\%) while, on average, the other data sets are somewhat higher: average ratios with Galavís et al. (1998), Tayal (2006b) and Aggarwal \& Keenan (2008a) are 1.06(4), 1.05(6) and $1.17(5)$, respectively. In this comparison we have corrected the $A$-value by Tayal (2006b) for the $2 \mathrm{p}^{3}{ }^{2} \mathrm{D}_{3 / 2}^{\mathrm{o}}-2 \mathrm{~s} 2 \mathrm{p}^{2}{ }^{2} \mathrm{P}_{1 / 2}$ transition which we believe was misprinted.

For allowed transitions with $\log A \geq 6$ involving excited configurations, the agreement between the data sets by Tachiev \& Froese Fischer (2000), Corrégé \& Hibbert (2004), Tayal (2006b) and Aggarwal \& Keenan (2008a) is within a few percent except for decay transitions from the $2 \mathrm{~s}^{2} 3 \mathrm{p}^{2} \mathrm{P}_{J}^{\mathrm{o}}$ levels where very large discrepancies are encountered in Tayal (2006b) and Aggarwal \& Keenan (2008a) which are difficult to explain (see Table A.4). Moreover, we are of the opinion that the differences in the radiative data computed by Aggarwal \& Keenan (2008a) for both allowed and forbidden transitions within the $n=2$ complex are not the result of the more formal treatment of the relativistic problem in the GRASP code, but perhaps due to limited configuration interaction.

Effective collision strengths for $\mathrm{O}$ IV have been computed with the $R$-matrix method by Blum \& Pradhan (1992) and Zhang et al. (1994) for transitions within $n=2$ and by Tayal (2006b) for 54 levels. On the other hand, Aggarwal \& Keenan (2008a) used the Dirac $R$-matrix DARC code to obtain rates for transitions among 75 levels. Both Tayal (2006b) and Aggarwal \& Keenan (2008a) have indicated 


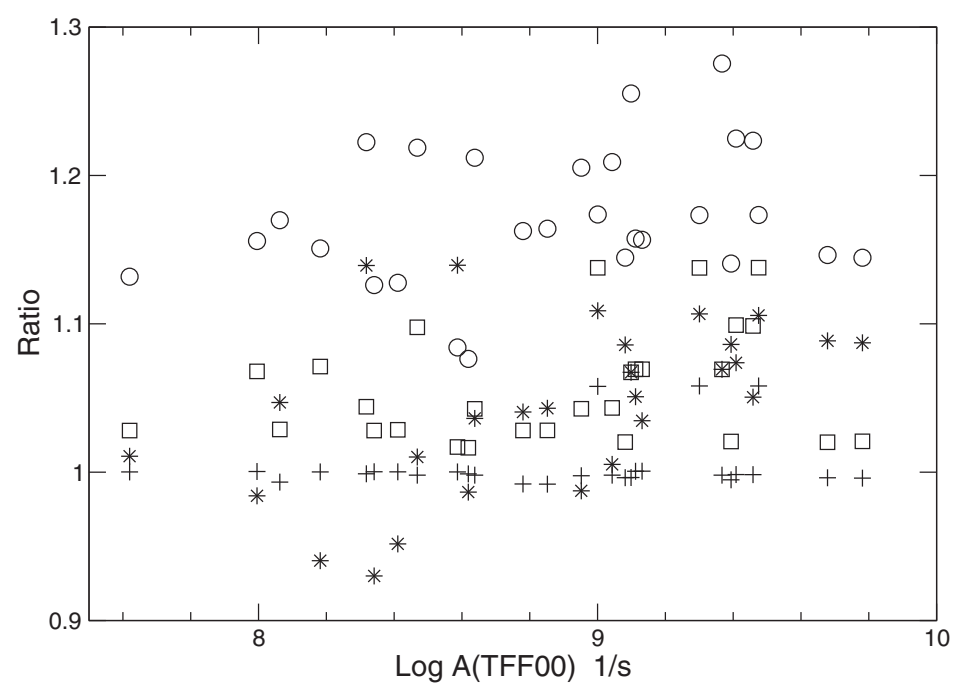

Fig. A.2. Ratios of $A$-values computed by Tachiev \& Froese Fischer (2000) for allowed transitions within the $n=2$ complex of O IV, A(TFF00), relative to: Galavís et al. (1998) (squares); Corrégé \& Hibbert (2004) (crosses); Tayal (2006b) (stars); and Aggarwal \& Keenan (2008a) (circles).

Table A.4. Discrepant $A$-values for E1 transitions in O IV.

\begin{tabular}{|c|c|c|c|c|c|}
\hline Upper level & Lower level & $\mathrm{TFF}$ & $\mathrm{CH}$ & $\mathrm{T}$ & $\mathrm{AK}$ \\
\hline $2 \mathrm{~s}^{2} 3 \mathrm{p}^{2} \mathrm{P}_{1 / 2}^{\mathrm{o}}$ & $2 \mathrm{~s} 2 \mathrm{p}^{2}{ }^{2} \mathrm{D}_{3 / 2}$ & & $4.87 \mathrm{E}+8$ & $5.09 \mathrm{E}+8$ & $8.20 \mathrm{E}+8$ \\
\hline $2 \mathrm{~s}^{2} 3 \mathrm{p}^{2} \mathrm{P}_{1 / 2}^{\mathrm{o}}$ & $2 \mathrm{~s} 2 \mathrm{p}^{2}{ }^{2} \mathrm{~S}_{1 / 2}$ & $9.31 \mathrm{E}+7$ & $1.03 \mathrm{E}+8$ & $5.33 \mathrm{E}+5$ & $1.48 \mathrm{E}+8$ \\
\hline $2 \mathrm{~s}^{2} 3 \mathrm{p}^{2} \mathrm{P}_{1 / 2}^{\mathrm{o}}$ & $2 \mathrm{~s} 2 \mathrm{p}^{2}{ }^{2} \mathrm{P}_{1 / 2}$ & $2.92 \mathrm{E}+6$ & $2.96 \mathrm{E}+6$ & $5.33 \mathrm{E}+5$ & $3.69 \mathrm{E}+6$ \\
\hline $2 \mathrm{~s}^{2} 3 \mathrm{p}^{2} \mathrm{P}_{1 / 2}^{\mathrm{o}}$ & $2 \mathrm{~s} 2 \mathrm{p}^{2}{ }^{2} \mathrm{P}_{3 / 2}$ & $1.14 \mathrm{E}+6$ & $1.14 \mathrm{E}+6$ & $4.17 \mathrm{E}+5$ & $1.47 \mathrm{E}+6$ \\
\hline $2 \mathrm{~s}^{2} 3 \mathrm{p}^{2} \mathrm{P}_{3 / 2}^{\mathrm{o}}$ & $2 \mathrm{~s} 2 \mathrm{p}^{2}{ }^{2} \mathrm{D}_{5 / 2}$ & $4.43 \mathrm{E}+8$ & $4.38 \mathrm{E}+8$ & $4.59 \mathrm{E}+8$ & $7.37 \mathrm{E}+8$ \\
\hline $2 \mathrm{~s}^{2} 3 \mathrm{p}^{2} \mathrm{P}_{3 / 2}^{\mathrm{o}}$ & $2 \mathrm{~s} 2 \mathrm{p}^{2}{ }^{2} \mathrm{D}_{3 / 2}$ & $4.92 \mathrm{E}+7$ & $4.86 \mathrm{E}+7$ & $5.15 \mathrm{E}+7$ & $8.18 \mathrm{E}+7$ \\
\hline $2 \mathrm{~s}^{2} 3 \mathrm{p}^{2} \mathrm{P}_{3 / 2}^{\mathrm{o}}$ & $2 \mathrm{~s} 2 \mathrm{p}^{2}{ }^{2} \mathrm{~S}_{1 / 2}$ & $9.38 \mathrm{E}+7$ & $1.03 \mathrm{E}+8$ & $1.35 \mathrm{E}+8$ & $1.49 \mathrm{E}+8$ \\
\hline $2 \mathrm{~s}^{2} 3 \mathrm{p}^{2} \mathrm{P}_{3 / 2}^{\mathrm{o}}$ & $2 \mathrm{~s} 2 \mathrm{p}^{2}{ }^{2} \mathrm{P}_{3 / 2}$ & $3.21 \mathrm{E}+6$ & $3.21 \mathrm{E}+6$ & $9.67 \mathrm{E}+5$ & $4.08 \mathrm{E}+6$ \\
\hline
\end{tabular}

TFF: Tachiev \& Froese Fischer (2000). CH: Corrégé \& Hibbert (2004). T: Tayal (2006b). AK: Aggarwal \& Keenan (2008a).

that some of the effective collision strengths by Blum \& Pradhan (1992) display anomalous increases with temperature which will not be discussed here as this calculation was corrected and superseded by Zhang et al. (1994). Aggarwal \& Keenan (2008a) mention that the main differences with the data by Zhang et al. (1994) are at the lower temperatures, and that they are due to an overestimate of their top-up procedure. We do not agree with this conclusion because a comparison of the two data sets at the high-temperature end, namely $\log T=5.7 \mathrm{~K}$, where 
convergence of the partial wave expansion is more conspicuous, results in satisfactory agreement (within 10-15\%). Discrepancies at the lower temperature end are mostly caused, as discussed previously, by the treatment of the resonance structure, energy meshes and target-level energy separations.

Aggarwal \& Keenan (2008a) find general overall accord with Tayal (2006b), the differences being mainly at low temperatures, but warn of extensive printing errors in his tables. In conclusion, we would recommend the effective collision strengths by Zhang et al. (1994) and Aggarwal \& Keenan (2008a) which are listed in 04.EColSt, particularly the latter due to the large number of transitions considered, and suggest that the data by Tayal (2006b) should be used with caution. The overall accuracy of these collisional data sets is estimated at $\sim 20 \%$.

\section{A.2.5 O V}

The 20-level atomic model we have selected for $\mathrm{O} \mathrm{v}$ has been computed with the MCHF code (Froese Fischer \& Tachiev 2004; Tachiev \& Froese Fischer 1999) including Breit-Pauli relativistic corrections and configuration interaction (see 05.Elev), which yields level energies that on average agree with experiment to around $0.1 \%$. We also tabulate level energies by Hibbert (1980) calculated with CIV3 in a similar approximation that are within $1 \%$.

Tachiev \& Froese Fischer (1999) tabulate $A$-values for both allowed (E1) and forbidden transitions (E2, M1, M2) which are compared in 05. Aval with data sets by Hibbert (1980) and Kingston \& Hibbert (2000, 2001). For E1 transitions within the $n=2$ complex, Hibbert (1980) and Kingston \& Hibbert (2001) agree with Tachiev \& Froese Fischer (1999) to better than $15 \%$ and $10 \%$, respectively, while for transitions to levels in the $2 \mathrm{~s}^{2} 3 l(l \leq 2)$ configurations, Hibbert $(1980)$ agrees to around $12 \%$. In this comparison some of the $A$-values by Hibbert (1980) have been corrected for misprints. For forbidden transitions among levels of the $1 \mathrm{~s}^{2} 2 \mathrm{~s}^{2}$ and $1 \mathrm{~s}^{2} 2 \mathrm{~s} 2 \mathrm{p}$ configurations, $A$-values by Kingston \& Hibbert (2000) agree with those by Tachiev \& Froese Fischer (1999) to better than 5\%. A measurement of the radiative lifetime of the $2 \mathrm{~s} 2 \mathrm{p}{ }^{3} \mathrm{P}_{1}^{\mathrm{o}}$ level in a heavy-ion storage ring by Träbert et al. (2005) at $0.432(9) \mathrm{ms}$ compares well with the theoretical values: $0.461 \mathrm{~ms}$ (Hibbert 1980); $0.437 \mathrm{~ms}$ (Tachiev \& Froese Fischer 1999) and $0.481 \mathrm{~ms}$ (Kingston \& Hibbert 2000).

Effective collision strengths for transitions among the 20 levels of the proposed atomic model have been computed by K.A. Berrington and A.E. Kingston but were never published although they have been parameterised by Kato, Lang, \& Berrington (1990). For these two reasons, we have chosen not to list them in the corresponding 05 spreadsheet. On the other hand, Smith et al. (2005) have measured absolute effective collision strengths for the $2 \mathrm{~s}^{2}{ }^{1} \mathrm{~S}-2 \mathrm{~s} 2 \mathrm{p}{ }^{1} \mathrm{P}^{\mathrm{o}}$ transition finding good agreement (better than 25\%) with theory thus providing a useful benchmark. 


\section{A.2.6 O VI}

For Li-like O vi we adopt the 24-level atomic model $\left(1 \mathrm{~s}^{2} n l\right.$ for $\left.n \leq 5\right)$ computed by Tayal (2003), Aggarwal \& Keenan (2004) and, more recently, by Liang \& Badnell (2011) with the CIV3, GRASP and AUTOSTRUCTURE atomic structure codes, respectively, where the theoretical energies agree with measurements to better than 1\% (see 06.Elev). Tayal (2003) and Aggarwal \& Keenan (2004) only list $A$-values for allowed lines while Liang \& Badnell (2011) include the forbidden transitions as well (06.Aval). We also compare with the $A$-values for allowed lines computed by Nahar (2002) with the Breit-Pauli $R$-matrix method. In general, $A$-values for allowed lines compare very well (within a few per cent) except for the $5 \mathrm{p}-3$ s transition where the $A$-value by Tayal (2003), $3.08 \times 10^{9} \mathrm{~s}^{-1}$, is notably larger than those by Nahar (2002), $1.81 \times 10^{9} \mathrm{~s}^{-1}$, Aggarwal \& Keenan (2004), $1.79 \times 10^{9} \mathrm{~s}^{-1}$, and Liang \& Badnell (2011), $1.75 \times 10^{9} \mathrm{~s}^{-1}$.

Effective collision strengths have been computed with the $R$-matrix method including pseudo-states (Tayal 2003), the DARC code (Aggarwal \& Keenan 2004) and, more recently, with the intermediate-coupling frame transformation (ICFT) $R$-matrix approach (Liang \& Badnell 2011). All these data sets are available from 06.EColSt. Liang \& Badnell (2011) include a target with both valenceshell $\left(1 \mathrm{~s}^{2} n l, n \leq 5\right)$ and inner-shell $\left(1 s 2 \ln l^{\prime}, n \leq 4\right)$ levels, and take into account radiative and Auger damping. We find very good general agreement between Tayal (2003), Aggarwal \& Keenan (2004) and Liang \& Badnell (2011) (around 10\%), except for some transitions in Tayal (2003) with small effective collision strengths at low temperatures $(\log T<4.5 \mathrm{~K})$ which are dominated by resonances, and for some allowed transitions for $\log T>6 \mathrm{~K}$ which are affected by partial wave convergence. This comparison also appears to indicate that, at least for valencevalence collisional transitions in $\mathrm{O}$ VI, damping effects on the resonance structures are still small. We confirm the reliability of these three collisional data sets.

\section{A.2.7 O VII}

Energy levels for He-like O viI have been computed by Delahaye \& Pradhan (2002) for 31 levels $(n \leq 4)$ with SUPERSTRUCTURE in a Breit-Pauli configurationinteraction method, and by Aggarwal \& Keenan (2008b) for 49 levels $(n \leq 5)$ with GRASP in a multi-configuration Dirac-Fock approach, the overall agreement with the spectroscopic measurements being better than $1 \%$. We adopt the atomic model by Aggarwal \& Keenan (2008b) in 07 .Elev.

Aggarwal \& Keenan (2008b) list $A$-values for both allowed and forbidden transitions with $n \leq 5$ while Delahaye \& Pradhan (2002) only do so for allowed transitions with $n \leq 3$ (see 07 .Aval). For transitions with $\log A \geq 7$, a comparison of these two data sets results in an agreement within 10\%. Furthermore, Crespo López-Urrutia et al. (1998) report a measured lifetime for the $1 \mathrm{~s} 2 \mathrm{~s}{ }^{3} \mathrm{~S}_{1}$ level of $0.956(5) \mathrm{ms}$ obtained with an electron beam ion trap (EBIT), which is in the same level of agreement with the theoretical estimate of $1.06 \mathrm{~ms}$ (Aggarwal \& Keenan 2008b). 
Effective collision strengths have been computed by Delahaye \& Pradhan (2002) in a Breit-Pauli $R$-matrix method taking into account radiative damping, and by Aggarwal \& Keenan (2008b) using the Dirac $R$-matrix DARC code. The latter have performed a detailed comparison of the two sets of rates which results in differences greater than $20 \%$ for most of the transitions listed by Delahaye \& Pradhan (2002). At the lower temperatures, where the effective collision strengths are sensitive to the resonance structures, discrepancies up to a factor of 2 are found but decrease significantly with temperature; furthermore, for levels $i \geq 23$ they can be as large as an order of magnitude. Since Delahaye \& Pradhan (2002) found the effects of radiative damping small in this system, such large differences are not readily explainable. As a result, we would recommend the data set by Aggarwal \& Keenan (2008b) which have been rated at better than $20 \%$ for most transitions and is available in 07 .EColst.

\section{A.2.8 O VIII}

Aggarwal et al. (2010) have recently computed radiative and collisional rates for H-like O VIII with a 25-level $(n \leq 5)$ atomic model. Level energies have been determined with the GRASP multi-configuration Dirac-Fock code with and without QED effects. C. Mendoza, A. Chechelev, P. Palmeri, P. Quinet, N.R. Badnell \& T.R. Kallman (private communication) have also used this code to generate level energies (including QED effects) and $A$-values for this system which are used here for comparison purposes. As shown in 08.Elev, theoretical energies agree with the spectroscopic values to $3-4$ parts in $10^{5}$ if QED corrections are taking into account.

Transition probabilities for both allowed and forbidden transitions are listed in 08. Aval, the main difference between the data sets by Aggarwal et al. (2010) and Mendoza et al. is that the latter include the electric (E3) and magnetic (M3) octupole channels which for some transitions, e.g. $n \mathrm{~d}_{5 / 2}-n^{\prime} \mathrm{p}_{1 / 2}$ and $n f_{5 / 2}-n^{\prime} \mathrm{s}_{1 / 2}$, are the dominant decay routes.

The effective collision strengths by Aggarwal et al. (2010) have been computed with the Dirac $R$-matrix code (DARC). Since there is no previous work to compare with, the relativistic, multi-configuration, distorted-wave Flexible Atomic Code (FAC) by Gu (2003) has been used by Aggarwal et al. (2010) to determine the accuracy level, finding significant resonance enhancements at the lower temperatures to arrive at an accuracy rating of better than $20 \%$.

\section{A.3 Optical recombination lines}

In low density plasmas, metal abundances can also be estimated from the analysis of optical recombination lines. This method, compared to the most common one based on collisionally excited lines, has the advantage that the line intensity ratios relative to hydrogen depend weakly on electron temperature while collisionally excited lines display emissivities that vary exponentially with temperature. 
The resulting abundances, however, have been found to be systematically larger: typically by a factor of 2 in $\mathrm{H}$ II regions and higher in most planetary nebulae (a factor of 70 has been reported, see Liu et al., 2006; Fang et al., 2011 and Chapter 2). In order to explain this dilemma, some authors, e.g. Liu et al. (2000), have proposed the existence of metal-rich, cold plasma inclusions in the diffuse gas. Others, namely Nahar et al. (2010), have suggested that dominant near-threshold resonance structures, such as those found in the photoionisation cross section of $\mathrm{O}$ II, could indeed increase the dielectronic recombination rates at very low temperatures so as to sort out the abundance anomaly.

Therefore, in this context it is important to rely outright on very well-resolved photoionisation cross sections and accurate effective recombination coefficients. In the unified method originally proposed by Storey (1994) for O II, the latter are determined directly from the photoionisation cross section of each state thus taking into account both radiative and dielectronic processes; in the case of $\mathrm{O}$ ions, extensive calculations have been performed in this approach by Nahar (1999, 2004).

Most of the work on the optical recombination lines have been carried out in the $L S$ coupling scheme, except perhaps for the intermediate-coupling treatment of O III recombination by Kisielius \& Storey (1999). As a consequence, a new set of very detailed calculations of effective recombination coefficients are currently being performed for N II (Fang et al. 2011) and O II (in progress) taking into account relativistic effects at very low temperatures $(\sim 100 \mathrm{~K})$. It is found that the sensitivity of the individual spectral term components to temperature and density can lead to new plasma diagnostics. In the expectation of this new work, we neither include recommended photoionisation cross sections nor recombination rates for $\mathrm{O}$ ions in our supplementary online material.

\section{A.4 Charge transfer}

In many astrophysical plasmas containing oxygen species (e.g. comets, planetary atmospheres and the heliosphere), charge transfer reactions can compete with radiative and dielectronic recombination in determining ionisation fractions (Chutjian 2004; Greenwood et al. 2004; Koutroumpa et al. 2007; Stancil 2001). They essentially involve inelastic collisions between heavy particles, namely atoms, molecules and ions, whereby $m$ electrons bound to a neutral target $(X)$ are transferred to the incoming O-ion projectile

$$
O^{q+}+X \rightarrow O^{(q-m)+}+X^{m+}
$$

$X$ is usually a hydrogen or helium atom (Stancil et al. 1999; Wu et al. 2009; Zhao et al. 2005), but $\mathrm{H}_{2}, \mathrm{H}_{2} \mathrm{O}, \mathrm{CO}, \mathrm{CO}_{2}$ or $\mathrm{CH}_{4}$ molecular targets may also be involved (Djurić et al. 2008; Greenwood et al. 2004; Hasan et al. 2001; Mawhorter et al. 2007). Moreover, although the reactants are usually in their ground states, the exchanged electron can recombine into an excited $n l$ state of $O^{(q-1)+}$ which then radiates (Dalgarno \& Sternberg 1982), and the inverse reactions where a neutral 
$\mathrm{O}$ target reacts with an ionic projectile such as $\mathrm{He}^{+}$can also take place (Zhao et al. 2004).

A keynote contribution in this field has been the comprehensive table of analytic fits to charge transfer rates for reactions between $\mathrm{H}$ and ions with $q=1-4$ and $Z \leq 30$ released by Kingdon \& Ferland (1996), where quantal rates were in many cases complemented with data computed with the Landau-Zener approximation of the earlier days of Butler et al. (1979).

As described by Wang et al. (2002), a variety of theoretical methods are currently used to compute total and state-selective charge exchange rates according to the nature of the projectile-target system and energy range of interest. Good agreement with measurements is often found (see, for instance, Wu et al., 2009), and online databases (e.g. the ORNL/UGA Charge Transfer Database for Astrophysics $\left.^{24}\right)$ are being developed.

\section{A.5 K-shell photoabsorption}

The diagnostic potential of oxygen K-shell photoabsorption is currently being exploited in the high-resolution X-ray spectra obtained with the Chandra and XMMNewton satellite-borne observatories. We are here concerned with inner-shell transitions in oxygen ions that give rise to spectral features such as $\mathrm{K} \alpha$ and $\mathrm{K} \beta$ lines and $\mathrm{K}$ edges that are observed in a variety of exotic astronomical entities: Seyfert galaxies, AGN dusty warm absorbers, black-hole candidates, black-hole binaries, bursting neutron stars and the warm-hot intergalactic medium, to mention a few (see García et al. 2005 and references therein).

Inner-shell absorption features in the X-ray spectrum of galactic sources are being employed to determine the oxygen abundances and ionisation fractions in the interstellar medium and to discern the phase of the absorbers, i.e. whether atomic or molecular, gas or solid (Juett et al. 2004). It is argued that the oscillatory modulations near the $\mathrm{K}$ edge due to the presence of solid particles (grains) can be detected (Lee et al. 2009), but in the case of the Sco X-1 low-mass X-ray binary, extensive spectral modelling by García et al. (2011) only found evidence of atomic oxygen.

In any case, the spectral modelling of oxygen K-shell photoabsorption requires accurate atomic data such as K-vacancy level energies, wavelengths, $f$-values, radiative and Auger widths and high-energy photoabsorption cross sections (see the OK spreadsheet). Although most of them are obtained through calculation, using atomic physics codes such as those mentioned in Section A.2, laboratory wavelengths for oxygen $\mathrm{K}$ lines have been measured by Schmidt et al. (2004) and Gu et al. (2005) in the high-resolution spectra of electron beam ion traps (EBIT). As shown in Table A.5, it is evident that the theoretical efforts will never match spectroscopic accuracy, but on the other hand, measurements may exhibit notorious misidentifications such as those by Gu et al. (2005) for O VI and O III.

\footnotetext{
${ }^{24}$ http://www-cfadc.phy.ornl.gov/astro/ps/data/
} 
Table A.5. Comparison of EBIT and theoretical wavelengths $(\AA)$ for O K lines.

\begin{tabular}{lllll}
\hline \hline Ion & Lower level $(J)$ & Upper level $\left(J^{\prime}\right)$ & EBIT & Theory \\
\hline O vI & $1 \mathrm{~s}^{2} 2 \mathrm{~s}(1 / 2)$ & $1 \mathrm{~s} 2 \mathrm{~s} 2 \mathrm{p}(1 / 2,3 / 2)$ & $22.0194(16)^{a}$ & $22.00^{c}$ \\
& & & $22.374(8)^{b}$ & $22.05^{d}$ \\
& & & & $22.03^{e}$ \\
O v & \multirow{2}{*}{$1 \mathrm{~s}^{2} 2 \mathrm{~s}^{2}(0)$} & & $22.374(3)^{a}$ & $22.33^{c}$ \\
& & & $22.370(10)^{b}$ & $22.35^{d}$ \\
& & & & $22.37^{e}$ \\
O IV & \multirow{2}{*}{$1 \mathrm{~s}^{2} 2 \mathrm{~s}^{2} 2 \mathrm{p}(1 / 2,3 / 2)$} & $1 \mathrm{~s} 2 \mathrm{~s}^{2} 2 \mathrm{p}^{2}(1 / 2,3 / 2)$ & $22.741(5)^{b}$ & $22.78^{c}$ \\
& & & & $22.73^{d}$ \\
& & & & $22.75^{e}$ \\
O III & \multirow{2}{*}{$1 \mathrm{~s}^{2} 2 \mathrm{~s}^{2} 2 \mathrm{p}^{2}(1,2)$} & $1 \mathrm{~s} 2 \mathrm{~s}^{2} 2 \mathrm{p}^{3}(1)$ & & $23.08^{c}$ \\
& & & & $23.05^{d}$ \\
& & & & $23.07^{e}$ \\
\hline
\end{tabular}

${ }^{a}$ EBIT measurement (Schmidt et al. 2004). ${ }^{b}$ EBIT measurement (Gu et al. 2005). ${ }^{c}$ HULLAC calculation (Behar \& Kahn 2002). ${ }^{d} R$-matrix calculation (Pradhan et al. 2003). ${ }^{e}$ HFR calculation (García et al. 2005).

The most interesting effect in K-shell photoabsorption is by far the smearing of the K-edge resonance structure by both radiative and Auger damping. When a photon promotes a K-shell electron to an excited Rydberg state

$$
h \nu+1 \mathrm{~s}^{2} 2 \mathrm{~s}^{\lambda} 2 \mathrm{p}^{\mu} \longrightarrow 1 \mathrm{~s} 2 \mathrm{~s}^{\lambda} 2 \mathrm{p}^{\mu} n \mathrm{p}
$$

it decays via the radiative and Auger manifold

$$
\begin{aligned}
1 \mathrm{~s} 2 \mathrm{~s}^{\lambda} 2 \mathrm{p}^{\mu} n \mathrm{p} & \stackrel{\mathrm{K} n}{\longrightarrow} 1 \mathrm{~s}^{2} 2 \mathrm{~s}^{\lambda} 2 \mathrm{p}^{\mu}+h \nu_{n} \\
& \stackrel{\mathrm{K} \alpha}{\longrightarrow} 1 \mathrm{~s}^{2} 2 \mathrm{~s}^{\lambda} 2 \mathrm{p}^{\mu-1} n \mathrm{p}+h \nu_{\alpha} \\
& \stackrel{\mathrm{KL} n}{\longrightarrow}\left\{\begin{array}{l}
1 \mathrm{~s}^{2} 2 \mathrm{~s}^{\lambda} 2 \mathrm{p}^{\mu-1}+e^{-} \\
1 \mathrm{~s}^{2} 2 \mathrm{~s}^{\lambda-1} 2 \mathrm{p}^{\mu}+e^{-}
\end{array}\right. \\
& \stackrel{\mathrm{KLL}}{\longrightarrow}\left\{\begin{array}{l}
1 \mathrm{~s}^{2} 2 \mathrm{~s}^{\lambda} 2 \mathrm{p}^{\mu-2} n \mathrm{p}+e^{-} \\
1 \mathrm{~s}^{2} 2 \mathrm{~s}^{\lambda-1} 2 \mathrm{p}^{\mu-1} n \mathrm{p}+e^{-} \\
1 \mathrm{~s}^{2} 2 \mathrm{p}^{\mu} n \mathrm{p}+e^{-}
\end{array}\right.
\end{aligned}
$$

The radiative branches are controlled, as indicated in Equations (A.6)-(A.7), by the $\mathrm{K} \alpha(2 \mathrm{p} \rightarrow 1 \mathrm{~s})$ and $\mathrm{K} \beta(\mathrm{K} 3,3 \mathrm{p} \rightarrow 1 \mathrm{~s})$ lines. Reaction (A.8) contains the participator Auger (KLn) channels, where the $n$ p outer electron is directly involved in the decay, while in the KLL channels (Eq. A.9) the $n$ p Rydberg electron remains a spectator. For $\mathrm{O}^{q+}$ ions with $0 \leq q \leq 5$, Auger decay dominates, and for $q \leq 4$, it is almost completely through the KLL channels causing the total widths of $\mathrm{K}$-vacancy resonances within each species to be approximately constant as $n \rightarrow \infty$, 

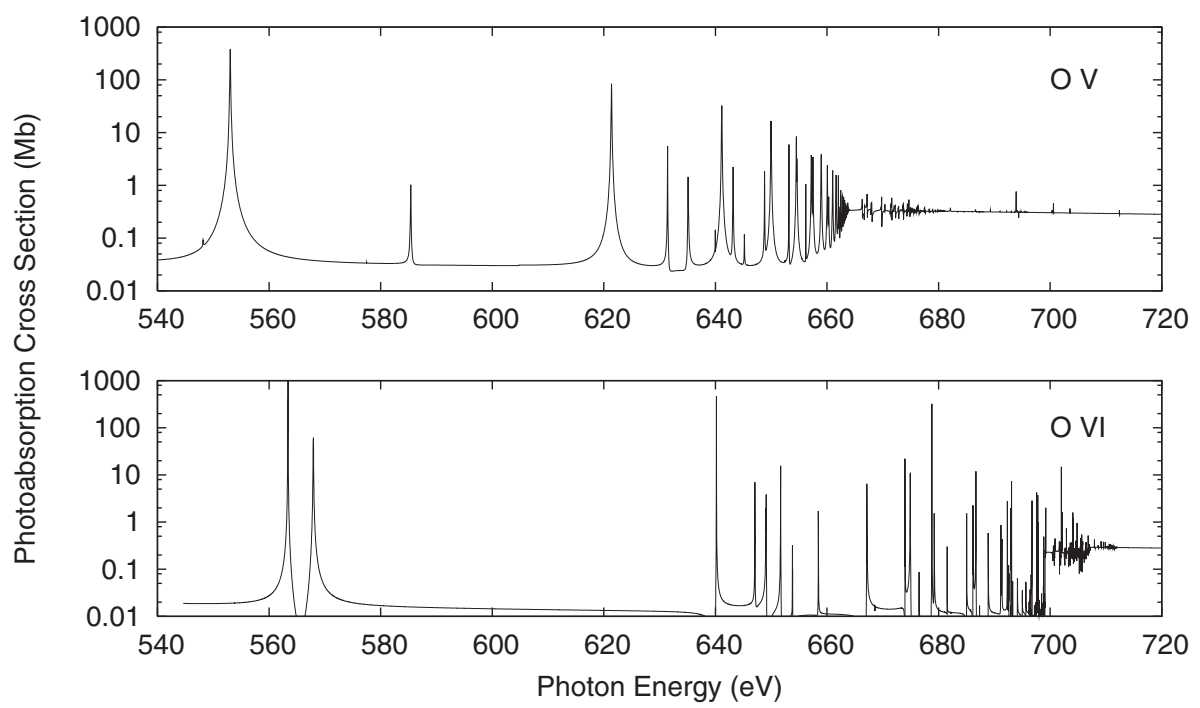

Fig. A.3. High-energy photoionisation cross section of $\mathrm{O} v$ (upper panel) showing the smearing of the $\mathrm{K}$ edge due to Auger damping. In $\mathrm{O}$ vi (lower panel), Auger damping does not occur thus exhibiting a sharp $\mathrm{K}$ edge. These cross sections are from the work by García et al. (2005).

thus imprinting distinctive signatures (Lorentzian profiles) on the threshold structures of the photoabsorption cross section (see Fig. A.3). This peculiar damping behaviour leads to the smearing of the $\mathrm{K}$ edge that has been corroborated in the laboratory (Gorczyca \& McLaughlin 2000), and its theoretical treatment requires the introduction of an optical potential in the $R$-matrix formalism (Gorczyca \& Robicheaux 1999).

\section{A.6 Opacities}

Rosseland mean opacities and radiative accelerations for oxygen computed by the Opacity Project can be currently retrieved online from the OPserver ${ }^{25}$ (Mendoza et al. 2007). They were last revised by Seaton \& Badnell (2004) and Badnell et al. (2005) particularly for inner-shell processes and by introducing an improved frequency mesh.

Since the OPAL Project (Rogers \& Iglesias 1992) did not make mean opacities for a pure oxygen mixture directly available, and due to the downward revision of the solar oxygen abundance (Asplund et al. 2004) and the important contribution of this element to the Rosseland mean at the base of the solar convection zone, Badnell et al. (2005) have explored several possibilities that could actually lead to

\footnotetext{
${ }^{25}$ http://opacities.osc.edu/
} 
enhancements of its opacity. However, contrary to the required increases of the Rosseland mean in the temperature region $2 \times 10^{6} \lesssim T \lesssim 5 \times 10^{6} \mathrm{~K}$ (Bahcall et al. 2005), they did not prove to be plausible.

New light-element opacities are currently being computed (J. Colgan, private communication) with the Los Alamos ATOMIC code (Magee et al. 2004), and members of the Opacity Project are also expected to revise and extend their data sets in the near future, particularly by introducing improved atomic models in intermediate coupling and accurate photoionisation cross sections for neutral and near-neutral species, and by considering in detail the iron-group elements. 
